\title{
EDITORIAL
}

\section{Heterogeneity of outcome with single-agent carfilzomib: all relapsed/refractory myelomas are not created equal}

\section{Leukemia (2013) 27, 2269-2271; doi:10.1038/leu.2013.161}

Since the year 2000, there has been a continued improvement in survival outcomes for multiple myeloma (MM) patients, ${ }^{1}$ attributable to the clinical development and inclusion of novel agents in upfront therapy, and improvement in high-dose therapy administration. $^{2}$ There has been a growing body of literature furthering the biological understanding of the disease. There is a clear recognition of both inter-patient and intra-patient genomic heterogeneity in multiple myeloma, ${ }^{3}$ although the academic community is still trying to comprehend specific phenotypic presentations such as primary plasma cell leukemia ${ }^{4}$ and extramedullary disease. ${ }^{5}$ Even with effective upfront therapeutic strategies, which seemingly help a greater proportion of MM patients achieve a 'complete' remission by biochemical and pathology criteria, relapses do occur sooner or later that require subsequent lines of therapy. There is a growing list of classes of novel agents that are disease-relevant and showing promise in early clinical investigations in the relapsed/refractory MM (RRMM) setting. These classes include histone deacytelase inhibitors, heatshock protein inhibitors, kinesin spindle protein inhibitors, immunetherapeutics and ubiquitin-proteasome pathway targeting agents. ${ }^{6}$ We have witnessed the Food and Drug Administration approval of two novel agents in the past 12 months, namely carfilzomib and pomalidomide, in the RRMM setting. It is highly likely that several novel agents will acquire Food and Drug Administration approval for RRMM over the next 5 years. The challenges in this setting would then be to determine which class of drugs may be more suitable for an individual patient, whether it would be better to sequence or combine different drug classes, and whether or not it would be possible to achieve 'functional cure' by making RRMM a chronic disease for most patients.

In the present issue, Jakubowiak et al. ${ }^{7}$ have attempted to tease out the impact of cytogenetic abnormalities (CA) on the response and outcome of patients treated on the single-agent carfilzomib phase II trial (PX-171-003-A1), where $73 \%$ of the patients were bortezomib refractory. High-risk cytogenetics were defined as per the IMWG criteria as a fluorescent in situ hybridization positive for del17p13, $t(4 ; 14)$ or $t(14 ; 16)$, or deletion 13 or hypodiploidy by metaphase cytogenetics. CA data were available in 229 of the 257 evaluable patients, of which 167 (72.9\%) were identified as standard risk and 62 (27.1\%) were identified as high-risk patients. Most high-risk CA occurred as single abnormality $(18.8 \%)$, with del17p13, t( $4 ; 14)$ and hypodiploidy being the most common ones $(13.1 \%, 7.9 \%$ and $7.9 \%$, respectively). The high-risk group was reported to have similar overall response rate to standard-risk group $(25.8 \%$ vs $24.6 \%$, respectively) but with lower depth and duration of response as depicted by the CBR rate $(30.7 \%$ vs $40.7 \%$, respectively), and DOR (5.6 vs 8.3 months, respectively). What was really fascinating to observe was that patients with isolated $\mathrm{t}(4 ; 14)$ had a remarkably higher overall response rate $63.6 \%$ with a median progression-free survival (PFS) of 4.5 months and overall survival (OS) of 15.8 months, number suggesting this group did at least as well, if not better, than the standard- risk group. It was also clear that del17p13 predicted for poor outcome either by itself or in combination with other abnormalities, including $\mathrm{t}(4 ; 14)$.

The pre-novel therapy era identified $t(4 ; 14)$, del17p13 (hallmark of p53 deletion) and 1q21 chromosome gains as poor prognostic markers. ${ }^{8}$ Among the cytogenetic abnormalities considered high risk today, there now appears evidence that the poor prognosis associated with chromosomal translocation $(4 ; 14)$ may be mitigated by the addition of bortezomib as part of induction and consolidation in newly diagnosed transplant-eligible patients. The HOVON-65/GMMG-HD4 phase III trial randomized patients to receive either vincristine-adriamycin-dexamethasone (VAD) or bortezomib-adriamycin-dexamethasone (PAD) as induction followed by thalidomide maintenance in the VAD arm, and bortezomib maintenance in the PAD arm. ${ }^{9}$ The 3-year OS for patients with $\mathrm{t}(4 ; 14)$ was superior in the bortezomib arm $(82 \%$ vs $55 \%, P=0.0003)$, similar trends were observed for deletion $7 p(83 \%$ vs $33 \%, P<0.0001)$. Similar trends have been reported by the Arkansas team in the Total Therapy (TT) program, where the poor risk associated with MS molecular subgroup of patients (comprising FGFR3 gene and/or MMSET gene overexpression) that was observed in TT2 trial (no bortezomib) was overcome by inclusion of bortezomib in $T T 3 A^{10}$ and $T T 3 B^{11}$ trials (Figure 1). It was also observed that low p53 gene expression in TT3A trial ${ }^{12}$ was associated with poor outcome only in the GEP70 low-risk patients. The IFM 2005- $01^{13}$ trial (bortezomib-dexamethasone versus VAD induction pre-ASCT) also reported a partial mitigation of poor prognosis conferred by $t(4 ; 14)$ on the bortezomibdexamethasone arm when compared with the VAD arm, but no respite was observed for patients with del17p13. The VISTA trial ${ }^{14}$ (phase III: melphalan-prednisone with or without bortezomib) reported no statistical difference based on cytogenetic features for response, PFS or OS in newly diagnosed transplant in-eligible patients receiving on the bortezomib arm; it would be worthwhile evaluating the outcomes in patients with $t(4: 14)$ alone.

Although these data are in the upfront setting, they suggest that certain drugs or drug classes could be preferentially employed for patients with specific cytogenetic abnormalities. In the context of the available data in the upfront setting, it may be reasonable to even suggest that with the use of bortezomib, $\mathrm{t}(4 ; 14)$ may now be considered at least 'intermediate risk', if not a standard-risk cytogenetic abnormality; del17p13, however remains a challenge that needs to be overcome. Even though Jakubowiak et al. ${ }^{7}$ observed statistically significant differences in PFS/OS between patients with or without the high-risk cytogenetic abnormalities in a heavily pretreated and bortezomib-refractory MM population, there is a clear advantage in outcome for patients with isolated $t(4 ; 14)$ within the 'high-risk' cytogenetics RRMM group, suggesting that the use of carfilzomib in such patients may render their outcome comparable to standard-risk patients. These findings, along with the recent reports of pomalidomide efficacy in lenalidomiderefractory RRMM patients, ${ }^{15}$ also speak against a blanket 'classswitch' sequencing strategy in RRMM. 
Complete Response Duration, MS vs. Other Subgroups, TT3a

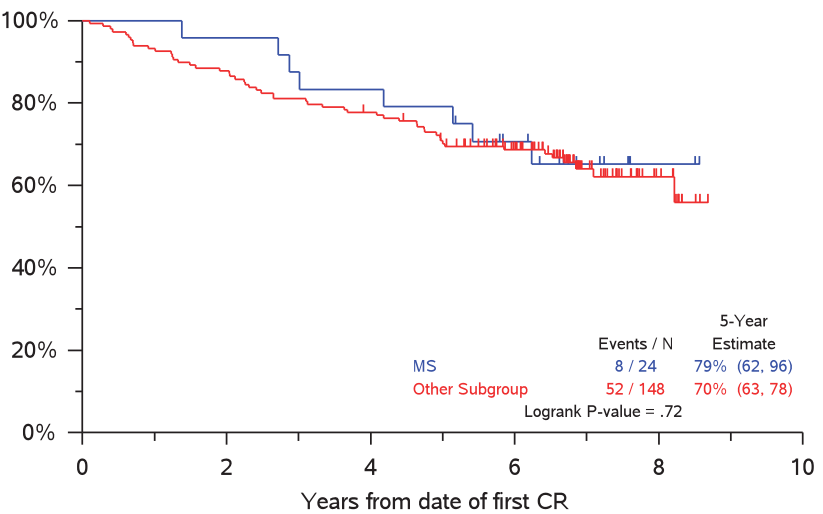

Overall Survival, MS vs. Other Subgroups, TT3a
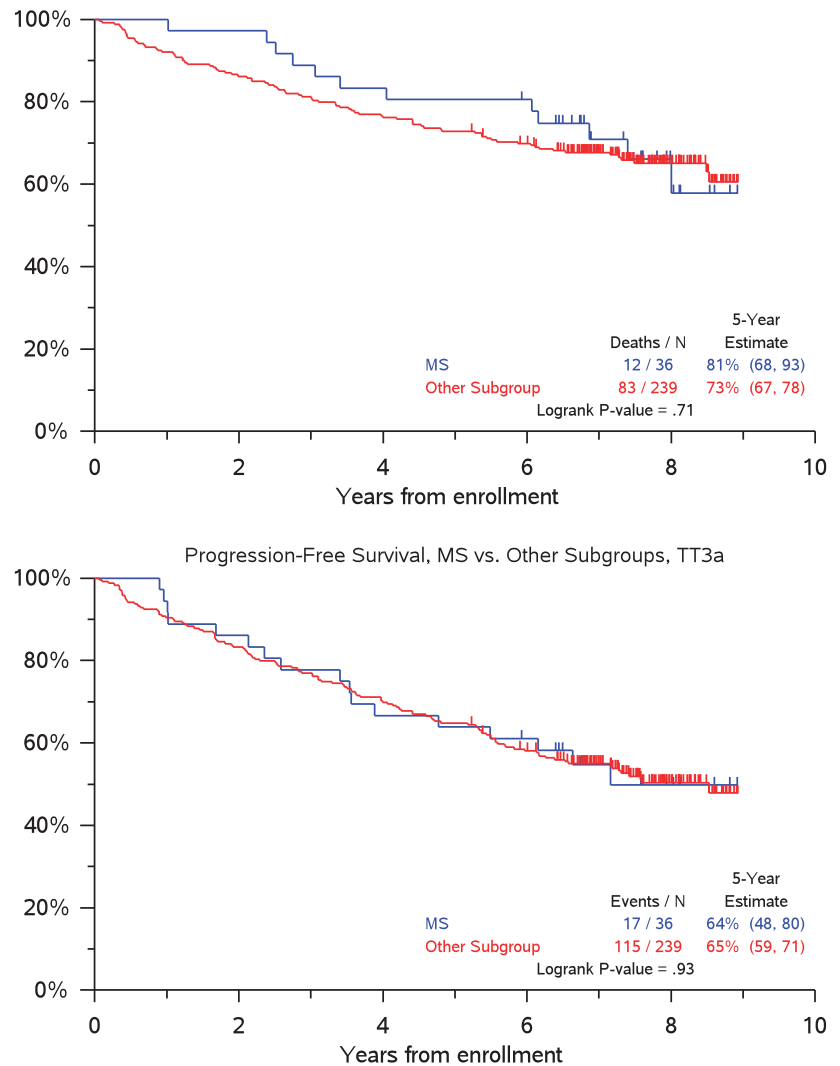

Complete Response Duration, MS vs. Other Subgroups, TT3b

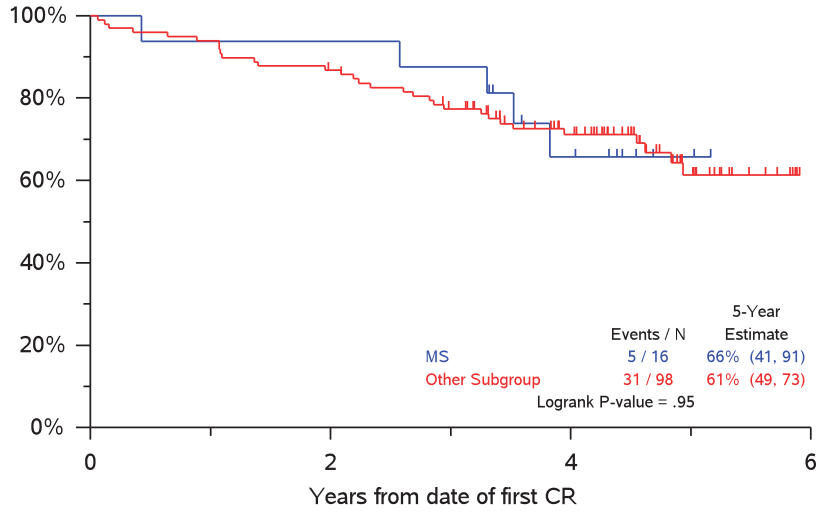

Overall Survival, MS vs. Other Subgroups, TT3b
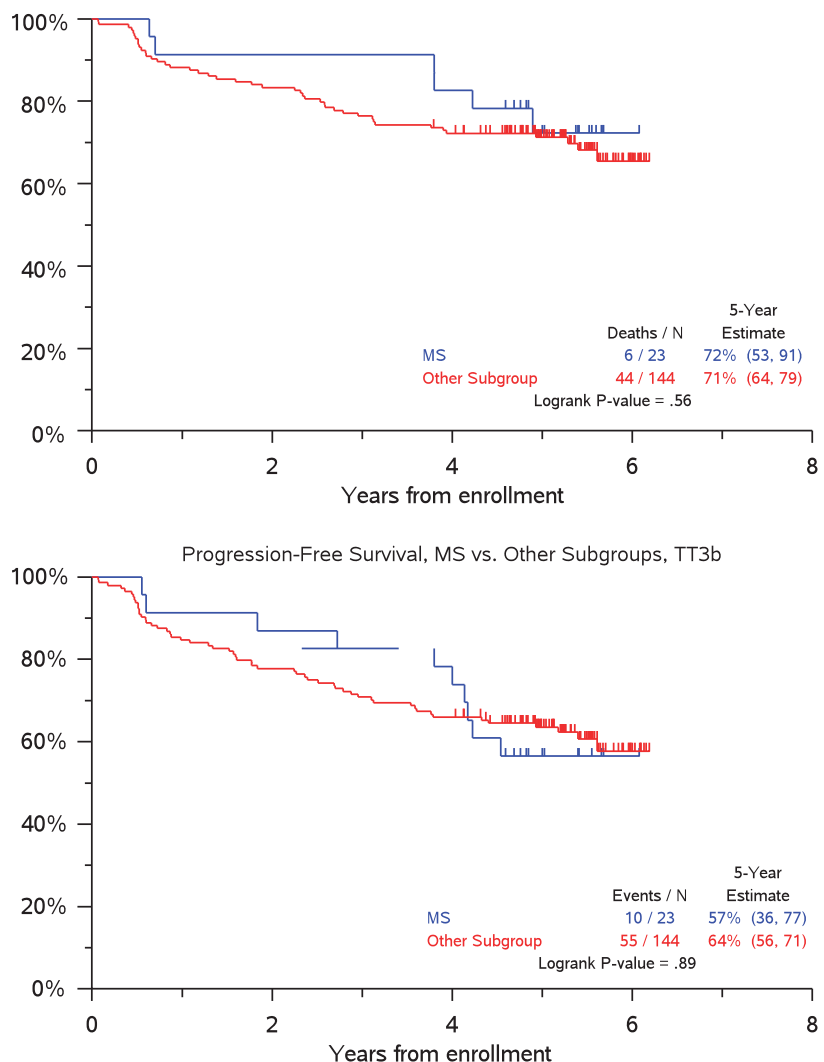

Figure 1. PFS, OS and complete response (CR) duration comparison between MS molecular subgroup and other subgroups in Total Therapy $3 \mathrm{~A}$ and $\mathrm{B}$ trials.

\section{CONFLICT OF INTEREST}

SZU is a consultant to Celgene, Millennium and Onyx. He has received research funding from Onyx and Celgene, and speaking honoraria from Celgene. AH does not have any conflict of interest to report.

SZ Usmani ${ }^{1}$ and A Hoering ${ }^{2}$

${ }^{1}$ Myeloma Institute for Research \& Therapy, University of Arkansas for Medical Sciences, Little Rock, AR, USA and

${ }^{2}$ Cancer Research and Biostatistics, Seattle, WA, USA

E-mail: susmani@uams.edu

\section{REFERENCES}

1 Kumar SK, Rajkumar SV, Dispenzieri A, Lacy MQ, Hayman SR, Buadi FK et al. Improved survival in multiple myeloma and the impact of novel therapies. Blood 2008; 111: 2516-2520.
2 Usmani SZ, Crowley J, Hoering A, Mitchell A, Waheed S, Nair B et al. Improvement in long-term outcomes with successive Total Therapy trials for multiple myeloma: are patients now being cured? Leukemia 2013; 27: 226-232.

3 Bahlis NJ. Darwinian evolution and tiding clones in multiple myeloma. Blood 2012; 120: 927-928.

4 Usmani SZ, Nair B, Qu P, Hansen E, Zhang Q, Petty N et al. Primary plasma cell leukemia: clinical and laboratory presentation, gene-expression profiling and clinical outcome with Total Therapy protocols. Leukemia 2012; 26: 2398-2405.

5 Usmani SZ, Heuck C, Mitchell A, Szymonifka J, Nair B, Hoering A et al. Extramedullary disease portends poor prognosis in multiple myeloma and is overrepresented in high-risk disease even in the era of novel agents. Haematologica 2012; 97: 1761-1767.

6 Lonial S, Mitsiades CS, Richardson PG. Treatment options for relapsed and refractory multiple myeloma. Clin Cancer Res 2011; 17: 1264-1277.

7 Jakubowiak AJ, Siegel DS, Martin T, Wang M, Viv R, Lanial S et al. Treatment outcomes in patients with relapsed and refractory multiple myeloma and 
high-risk cytogenetics receiving single-agent carfilzomib in the PX-171-003-A1 study. Leukemia 2013; 27: 2351-2356.

8 Avet-Loiseau H, Attal M, Campion L, Caillot D, Hulin C, Marit G et al. Long-term analysis of the IFM 99 trials for myeloma: cytogenetic abnormalities [t(4;14), del(17p), 1q gains] play a major role in defining long-term survival. J Clin Oncol 2012; 30: 1949-1952.

9 Neben K, Lokhorst HM, Jauch A, Bertsch U, Hielscher T, van der Holt B et al. Administration of bortezomib before and after autologous stem cell transplantation improves outcome in multiple myeloma patients with deletion 17p. Blood 2012; 119: 940-948.

10 Barlogie B, Anaissie E, van Rhee F, Haessler J, Hollmig K, Pineda-Roman M et al. Incorporating bortezomib into upfront treatment for multiple myeloma: early results of total therapy 3. Br J Haematol 2007; 138: 176-185.

11 Nair B, van Rhee F, Shaughnessy Jr JD, Anaissie E, Szymonifka J, Hoering A et al. Superior results of Total Therapy 3 (2003-33) in gene expression profiling-defined low-risk multiple myeloma confirmed in subsequent trial 2006-66 with VRD maintenance. Blood 2010; 115: 4168-4173.
12 Shaughnessy JD, Zhou Y, Haessler J, van Rhee F, Anaissie E, Nair B et al. TP53 deletion is not an adverse feature in multiple myeloma treated with total therapy 3. Br J Haematol 2009; 147: 347-351.

13 Avet-Loiseau H, Leleu X, Roussel M, Moreau P, Guerin-Charbonnel C, Caillot D et al. Bortezomib plus dexamethasone induction improves outcome of patients with $\mathrm{t}(4 ; 14)$ myeloma but not outcome of patients with del(17p). J Clin Oncol 28: 4630-4634.

14 San Miguel JF, Schlag R, Khuageva NK, Dimopoulos MA, Shpilberg O, Kropff M et al. Bortezomib plus melphalan and prednisone for initial treatment of multiple myeloma. N Engl J Med 2008; 359: 906-917.

15 Dimopolous M, Lacy M, Moreau P, Weisel KC, Song KW, Delforge M et al. Pomalidomide in Combination with Low-Dose Dexamethasone: Demonstrates a Significant Progression Free Survival and Overall Survival Advantage, in Relapsed/ Refractory MM: A Phase 3, Multicenter, Randomized, Open-Label Study. ASH 2012 Annual Meeting: LBA-6. 